\section{Persistent entanglement in three-level atomic systems}

To cite this article: M Ali Can et al 2004 J. Opt. B: Quantum Semiclass. Opt. 6 S13

View the article online for updates and enhancements.

\section{Related content}

- Transferring a cavity field entangled state
$\frac{\text { in cavity QED }}{\text { Liu Ye and Guang-Can Guo }}$
- Generating an entangled coherent state of
$\frac{\text { two cavity modes in a three-level Lambda- }}{\text { type atomic system }}$
Qing-Xia Mu, Yong-Hong Ma and L Zhou
- Entanglement, local measurements and
$\frac{\text { symmetry }}{\text { A A Klyachko and A S Shumovsky }}$

\section{Recent citations}

\begin{tabular}{l} 
- Entanglement in a system of a three-level \\
atom interacting with a single-mode field in \\
$\frac{\text { the presence of arbitrary forms of the }}{\text { nonlinearity and of the atomic initial state }}$ \\
\hline A-S F Obada et al \\
- Entanglement of a multi-photon three-level \\
$\frac{\text { atom interacting with a single-mode field in }}{\text { the presence of nonlinearities }}$ \\
A. -S. F. Obada et al \\
- Entanglement observation among single \\
mode bosonic field and an atom \\
N. Behzadi
\end{tabular} 


\title{
Persistent entanglement in three-level atomic systems
}

\author{
M Ali Can, Özgür Çakir, Alexander Klyachko and \\ Alexander Shumovsky
}

Faculty of Science, Bilkent University, Bilkent, Ankara, 06533, Turkey

E-mail: cakir@fen.bilkent.edu.tr

Received 24 June 2003, accepted for publication 1 August 2003

Published 5 March 2004

Online at stacks.iop.org/JOptB/6/S13 (DOI: 10.1088/1464-4266/6/3/003)

\begin{abstract}
We discuss the evolution towards persistent entangled state in an atom-photon system. A maximally entangled state can be stabilized at a local minimum of the system by draining some energy, thus obtaining a persistent entangled state. This scheme can be realized in three-level, $\Lambda$ type atomic systems since the third level is a meta-stable state. In particular, we compare dynamical description based on the exact and effective models. Some experimental realizations are discussed.
\end{abstract}

Keywords: entanglement, Jaynes-Cummings model, Markov approximation, three-level atom

This paper reports some new results relating to the entanglement in atomic systems. It builds upon our earlier investigations [1-3].

The generation and manipulation of entangled states in atom-photon systems has recently attracted a great deal of interest in the context of quantum information processing and quantum computing [4-10]. Most studies on entanglement in atomic systems have used the two-level atoms, interacting with photons via dipole transitions (e.g., see [11-13] and references therein). In this case, the lifetime of maximum entangled state (MES) in atomic subsystem is chiefly determined by the life of excited atomic state, that is by the natural line breadth. Usually, this time is quite short $[14,15]$. At the same time, the quantum information processing needs more or less durable entanglement.

According to the result obtained in [1], the maximum entanglement in a system corresponds to the maximum total local variance, describing the quantum fluctuations of all measurements. Thus, to achieve a long-lived maximum entanglement, we should first prepare a state with maximum quantum fluctuations and then stabilize it by draining energy right up to a (local) minimum, conserving at the same time the level of quantum fluctuations. This can be done via an interaction with a proper environment.

It was noticed in $[1,2]$ that the lifetime of atomic entanglement can be improved by entangling atoms with respect to the two atomic states, between which the dipole transition is forbidden. Besides that, since the system is

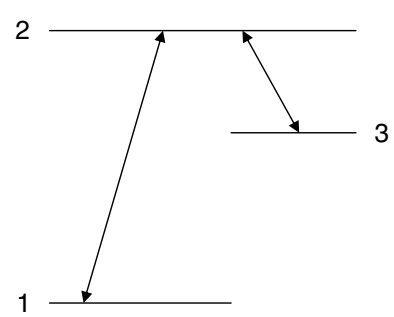

Figure 1. Scheme of three level $\Lambda$ type atomic configuration.

initially disentangled, to achieve the persistent entanglement an irreversible evolution of the system should be realized.

An important example is provided by the so-called $\Lambda$-type three-level structure $[16,17]$, which is illustrated in figure 1. Here the levels 1 and 2 are coupled by dipole transition as well as the levels 2 and 3 . Then, because of the selection rules with respect to parity, dipole transition between the levels 3 and 1 is forbidden [14]. The level 3 can be populated by a sort of Raman process, when atom first absorbs a pumping photon by $1 \rightarrow 2$ transition and then emits Stokes photon by $2 \rightarrow 3$ transition. If Stokes photon is then discarded, the atom will stay in the state 3 for a long time determined by either multipole or non-radiative processes.

Elimination of Stokes photon can be caused by a number of physical processes, involving dissipative environment. For example, in the case of atom in a cavity, Stokes photon can be absorbed by the cavity walls. Another possibility is 
provided by a two-mode cavity that is resonant with respect to the pumping mode and transparent for Stokes photons. In the former case, Stokes photons interact with continuum of 'phonon' modes, describing the standard HeisenbergLangevin mechanism of cavity losses [18, 19]. In the latter case, atoms interact with continuum of modes, corresponding to the natural line width for the level 2 .

Following [1-3], we can assume now that there are two identical three-level atoms in a cavity. The atoms are supposed to be prepared initially both in the ground state 1 , while the cavity contains a single pump photon. Then, the irreversible evolution caused by either of above mentioned processes will lead to the following atomic state

$$
\frac{1}{\sqrt{2}}(|3,1\rangle+|1,3\rangle)
$$

that definitely manifests maximum entanglement. The process of evolution towards the state (1) can be described through the use of two models. Within the first model, the interaction part of the Hamiltonian has the form

$$
H_{\text {int }}=\sum_{f}\left[\lambda_{\mathrm{P}} R_{21}(f) a_{\mathrm{P}}+\lambda_{\mathrm{S}} R_{23}(f) a_{\mathrm{S}}\right]+\text { H.c. },
$$

where $a_{\mathrm{P}}, a_{\mathrm{S}}$ are the annihilation operators of pumping and Stokes photons, $R_{i j}=|i\rangle\langle j|$ is the atomic transition operator, $f$ marks the atom, and $\lambda_{\mathrm{P}}, \lambda_{\mathrm{S}}$ are the coupling constants.

Instead of the one-photon three-level process described by the Hamiltonian (2), an effective model of two-photon process in two-level atom can also be considered [20,21]. In this case, it is assumed that the cavity is tuned consistent with two-photon energy conservation, i.e.

$$
E_{3}-E_{1} \approx \omega_{\mathrm{P}}-\omega_{\mathrm{S}},
$$

where $E_{i}$ denotes the energy of atomic level $i$. In this case, only one detuning parameter

$$
\Delta=E_{2}-E_{1}-\omega_{\mathrm{P}}=E_{2}-E_{3}-\omega_{\mathrm{S}}
$$

can be taken into account. Under the condition $\Delta \gg E_{3}-E_{1}$ the level 2 can be adiabatically eliminated [20], so that the dynamics of the system can be described by the effective interaction Hamiltonian of the form

$$
H_{\text {int }}^{\prime}=\sum_{f} \lambda R_{31} a_{\mathrm{S}}^{+} a_{\mathrm{P}}+\text { H.c. }
$$

where $\lambda=2 \lambda_{\mathrm{S}} \lambda_{\mathrm{P}} / \Delta$ is a certain effective coupling constant. The effective scheme of transitions is shown in figure 2 .

The two main objectives of this paper are on the one hand to show that both models describe the deterministic evolution towards the state (1), and on the other hand to ascertain the difference in the evolution process caused by the specific structure of the models.

Assume that the two identical atoms $(f=1,2)$ are located in a cavity that has quite high quality with respect to pumping mode $\omega_{\mathrm{P}}$, while absorb easily Stokes photons with $\omega_{\mathrm{S}}$. We assume that interatomic distance is much less than the wavelength of pumping and Stokes fields, so that the intracavity registration of Stokes photon cannot be used to identify

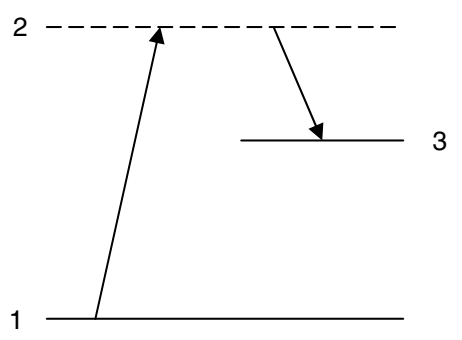

Figure 2. Effective scheme in the existence of strong detuning.

which atom is the source of this radiation. Consider first the complete model with the Hamiltonian

$$
\begin{gathered}
H=H_{0}+H_{\mathrm{loss}}+H_{\mathrm{int}}, \\
H_{0}=\omega_{\mathrm{P}} a_{\mathrm{P}}^{+} a_{\mathrm{P}}+\omega_{\mathrm{S}} a_{\mathrm{S}}^{+} a_{\mathrm{S}}+\sum_{f}\left(\omega_{21} R_{22}(f)+\omega_{31} R_{33}(f)\right), \\
H_{\mathrm{loss}}=\sum_{q} \eta_{q}\left(b_{q}^{+} a_{\mathrm{S}}+a_{\mathrm{S}}^{+} b_{q}\right)+\sum_{q} \Omega_{q} b_{q}^{+} b_{q},
\end{gathered}
$$

where $H_{\text {int }}$ coincides with (2), $\omega_{21}=E_{2}-E_{1}, \omega_{31}=E_{3}-E_{1}$, and $b_{q}^{+}$and $b_{q}$ are the Bose operators of 'phonons' in the cavity walls $[18,19]$.

With the total Hamiltonian (4) in hand, we can now write master equation for the reduced density matrix for the atomfield system that is obtained through the use of the so-called dressed-atom approximation (e.g., see [22-25]):

$\dot{\rho}=-\mathrm{i}\left[H_{0}+H_{\text {int }}, \rho\right]+\kappa\left\{2 a_{\mathrm{S}} \rho a_{\mathrm{S}}^{+}-a_{\mathrm{S}}^{+} a_{\mathrm{S}} \rho-\rho a_{\mathrm{S}}^{+} a_{\mathrm{S}}\right\}$.

Here $\rho$ denotes the density matrix and $\kappa$ is a physical quantity such that $\kappa^{-1}$ determines the lifetime of Stokes photon in the cavity and $Q=\omega_{23} / \kappa$ is the cavity quality factor with respect to Stokes photons.

Assume that the system is initially prepared in the state

$$
\left|\psi_{1}\right\rangle=|1,1\rangle \otimes\left|1_{P}, 0_{S}\right\rangle,
$$

so that both atoms are in the ground state 1 and cavity contains single pumping photon. Then, the evolution described by (5) takes place in the Hilbert space $\mathbb{H}$ spanned by the states (6) and

$$
\begin{aligned}
& \left|\psi_{2}\right\rangle=\frac{1}{\sqrt{2}}(|1,2\rangle+|2,1\rangle) \otimes\left|0_{\mathrm{P}}, 0_{\mathrm{S}}\right\rangle \\
& \left|\psi_{3}\right\rangle=\frac{1}{\sqrt{2}}(|1,3\rangle+|3,1\rangle) \otimes\left|0_{\mathrm{P}}, 1_{\mathrm{S}}\right\rangle \\
& \left|\psi_{4}\right\rangle=\frac{1}{\sqrt{2}}(|1,3\rangle+|3,1\rangle) \otimes\left|0_{\mathrm{P}}, 0_{\mathrm{S}}\right\rangle .
\end{aligned}
$$

Here $\left|\psi_{2}\right\rangle$ gives an intermediate entangled atomic state with short lifetime determined by the dipole decay either to the initial state $\left|\psi_{1}\right\rangle(6)$ or to another entangled state $\left|\psi_{3}\right\rangle$. In turn, absorption of Stokes photon by cavity walls lead to irreducible evolution from $\left|\psi_{3}\right\rangle$ to $\left|\psi_{4}\right\rangle$ that corresponds to the persistent atomic entangled state (1). Then, the density matrix in $\mathbb{H}$ takes the form

$$
\rho(t)=\sum_{j, \ell} \rho_{j \ell}(t)\left|\psi_{j}\right\rangle\left\langle\psi_{\ell}\right|, \quad j, \ell=1,2,3,4 .
$$

Use of (8) allows equation (5) to be cast into the form of sixteen differential equations that can be analysed numerically 


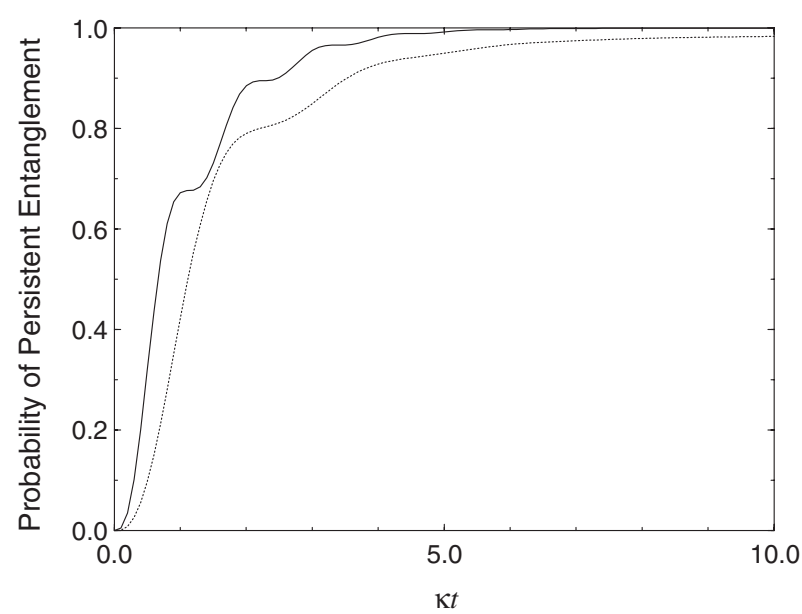

Figure 3. Evolution to the persistent entangled state in complete model (dotted curve) and in effective model (solid curve) for $\kappa=0.1 \lambda_{\mathrm{P}}, \Delta_{\mathrm{P}}=\Delta_{\mathrm{S}}=10 \lambda_{\mathrm{P}}, \lambda_{\mathrm{S}}=\lambda_{\mathrm{P}}$.

at different values of parameters. The results are shown in figure 3. It is seen that always

$$
\rho_{j \ell}(t) \rightarrow \begin{cases}1, & \text { at } j=\ell=4 \\ 0, & \text { otherwise }\end{cases}
$$

at $t \gg \kappa^{-1}$. Thus, the system described by the Hamiltonian (4) and prepared initially in the state (6) evolves towards the persistent entangled atomic state (1) without failure.

It is seen in figure 3 that evolution $\rho_{44} \rightarrow 1$ is not described by a smooth curve but has a stairs-like structure that becomes more visible with increase of $\kappa$. This specific behaviour is caused by the competition between the processes on transitions $1 \leftrightarrow 2$ and $2 \leftrightarrow 3$.

Consider now dynamics of the system within the framework of effective model with adiabatically eliminated excited level 2. Then, in the Hamiltonian (4), the interaction Hamiltonian $H_{\text {int }}^{\prime}$ (3) should be substituted instead of $H_{\text {int }}$ (2) and

$$
H_{0} \rightarrow H_{0}^{\prime}=\omega_{\mathrm{P}} a_{\mathrm{P}}^{+} a_{\mathrm{P}}+\omega_{\mathrm{S}} a_{\mathrm{S}}^{+} a_{\mathrm{S}}+\sum_{f} \omega_{31} R_{33}(f) .
$$

The master equation (5) can be used again with the same form of the Liouville term but with the change

$$
H_{0}+H_{\mathrm{int}} \rightarrow H_{0}^{\prime}+H_{\mathrm{int}}^{\prime}
$$

Since the level 2 is omitted, the state $\left|\psi_{2}\right\rangle$ in (7) should be discarded. Thus, the density matrix involves the states $\left|\psi_{1}\right\rangle$, $\left|\psi_{3}\right\rangle$ and $\left|\psi_{4}\right\rangle$ and consists of only nine elements instead of sixteen in previous case that enables us to fairly simplify the numerical analysis.

It can be seen again that the system evolves towards the persistent entangled state (1). At the same time, the absence of the contribution coming from the transition $1 \leftrightarrow 2$ leads to a more rapid evolution at the same $\kappa$ (figures 3,4 ).

Consider now the case of two-mode cavity, such that Stokes photons can leave it freely, while pumping-mode excitations exist for a very long time. Let us again begin with the one-photon three-level model. Then, the interaction

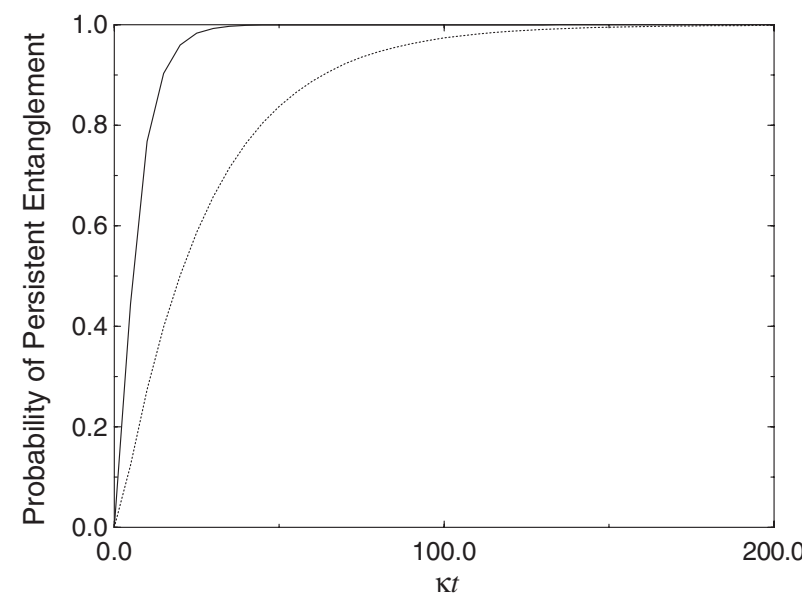

Figure 4. Evolution to the persistent entangled state in complete model (dotted curve) and in effective model (solid curve) for $\kappa=\lambda_{\mathrm{S}}=\lambda_{\mathrm{P}}, \Delta_{\mathrm{P}}=\Delta_{\mathrm{S}}=10 \lambda_{\mathrm{P}}$.

Hamiltonian (2) should be changed by

$$
H_{\text {int }}^{\prime \prime}=\sum_{f}\left[\lambda_{\mathrm{P}} R_{21}(f) a_{\mathrm{P}}+\sum_{k} \lambda_{\mathrm{S} k} R_{23}(f) a_{\mathrm{S} k}\right]+\text { H.c., }
$$

where summation over wavenumber $k$ corresponds to the natural line breadth of the level 2 with respect to the transition $2 \leftrightarrow 3$. In turn, the unperturbed part of the Hamiltonian should be chosen as follows

$$
\begin{aligned}
H_{0}^{\prime \prime} & =\omega_{\mathrm{P}} a_{\mathrm{P}}^{+} a_{\mathrm{P}}+\sum_{k} \omega_{\mathrm{S} k} a_{\mathrm{S} k}^{+} a_{\mathrm{S} k} \\
& +\sum_{f}\left[\omega_{21} R_{22}(f)+\omega_{31} R_{33}(f)\right] .
\end{aligned}
$$

The Hilbert space of the system with the Hamiltonian

$$
H^{\prime \prime}=H_{0}^{\prime \prime}+H_{\mathrm{int}}^{\prime \prime}
$$

is spanned by the vectors $\left|\psi_{1}\right\rangle(6),\left|\psi_{2}\right\rangle$ in (7), and

$$
\left|\psi_{3 k}\right\rangle=\frac{1}{\sqrt{2}}(|1,3\rangle+|3,1\rangle) \otimes\left|0_{\mathrm{P}}, 1_{\mathrm{S} k}\right\rangle,
$$

forming an infinite dimensional subspace.

The time-dependent wavefunction of the system (10) has the form

$$
|\Psi(t)\rangle=C_{1}(t)\left|\psi_{1}\right\rangle+C_{2}(t)\left|\psi_{2}\right\rangle+\sum_{k} C_{3 k}(t)\left|\psi_{3 k}\right\rangle
$$

with the initial conditions

$$
C_{\ell}(0)= \begin{cases}1, & \text { if } \ell=1 \\ 0, & \text { otherwise. }\end{cases}
$$

Then, the Schrödinger equation

$$
\mathrm{i} \frac{\partial}{\partial t}|\Psi(t)\rangle=H^{\prime \prime}|\Psi(t)\rangle
$$

with the Hamiltonian $H^{\prime \prime}$ leads to the following set of linear differential equations

$$
\begin{gathered}
\mathrm{i} \dot{C}_{1}=\omega_{\mathrm{P}} C_{1}+\lambda_{\mathrm{P}} \sqrt{2} C_{2} \\
\mathrm{i} \dot{C}_{2}=\omega_{21} C_{2}+\lambda_{\mathrm{P}} \sqrt{2} C_{1}+\sum_{k} \lambda_{\mathrm{S} k} C_{3 k} \\
\mathrm{i} \dot{C}_{3 k}=\left(\omega_{31}+\omega_{\mathrm{S} k}\right) C_{3 k}+\lambda_{\mathrm{S} k} C_{2} .
\end{gathered}
$$


To find solution of (14), let us express $C_{3 k}$ in terms of $C_{2}$ :

$$
C_{3 k}(t)=-\mathrm{i} \lambda_{\mathrm{S} k} \int_{0}^{t} C_{2}(\tau) \mathrm{e}^{\mathrm{i}\left(\omega_{31}+\omega_{\mathrm{Sk}}\right)(\tau-t)} \mathrm{d} \tau,
$$

convert summation over $k$ into an integration over continuum of Stokes modes corresponding to the natural line breadth of the level 2, and use Markovian approximation [19]. Then, the irreversible dynamics of the system is described by the two equations

$$
\begin{gathered}
\dot{C}_{1}=-\mathrm{i} \omega_{\mathrm{P}} C_{1}-\mathrm{i} \lambda_{\mathrm{P}} \sqrt{2} C_{2} \\
\dot{C}_{2}=-\mathrm{i} \omega_{21} C_{2}-\mathrm{i} \lambda_{\mathrm{P}} \sqrt{2} C_{1}-\Gamma C_{2},
\end{gathered}
$$

where $\Gamma=\pi \rho\left(\omega_{23}\right)\left|\lambda_{\mathrm{S} k}\right|^{2}\left(k=\omega_{23} / c\right)$ is the spontaneous decay rate for the transition $2 \rightarrow 3$ and $\rho\left(\omega_{23}\right)$ denotes the density of states of Stokes photons.

To within the second order in $\left|\lambda_{\mathrm{P}} / Z\right|$, where $Z=\Gamma-\mathrm{i} \Delta_{\mathrm{P}}$ and $\Delta_{\mathrm{P}}=\omega_{\mathrm{P}}-\omega_{12}$ is the detuning for the pumping mode, we get

$$
\begin{gathered}
C_{1}(t) \approx \mathrm{e}^{-\mathrm{i} \omega_{\mathrm{P}} t}\left[-\frac{2 \lambda_{\mathrm{P}}^{2}}{Z^{2}} \mathrm{e}^{-Z t}+\frac{Z^{2}-2 \lambda_{\mathrm{P}}^{2}}{Z^{2}} \mathrm{e}^{-\frac{2 \lambda_{\mathrm{P}}^{2} t}{Z}}\right], \\
C_{2} \approx-\frac{\lambda_{\mathrm{P}} \sqrt{2}}{\mathrm{i} Z}\left[\mathrm{e}^{-\Gamma t}-\mathrm{e}^{\frac{2 \lambda_{\mathrm{P}}^{2}+i Z \Delta_{\mathrm{P}}}{Z} t}\right] \mathrm{e}^{-\mathrm{i} \omega_{12} t} .
\end{gathered}
$$

The above approximations are reasonable because $\Gamma \gg$ $\lambda_{\mathrm{P}}, \lambda_{\mathrm{S} k}$.

In view of equation (12), the probability to achieve the states, corresponding to the persistent entanglement (1), is

$$
\mathcal{P}(t)=\sum_{k}\left|C_{3 k}(t)\right|^{2}=1-\left|C_{1}(t)\right|^{2}-\left|C_{2}(t)\right|^{2} .
$$

Since equations (16) and (17) describe the damped oscillations, we get

$$
\lim _{t \rightarrow \infty} \mathcal{P}(t)=1
$$

The detailed evolution of the probability (18) is shown in figure 5. It is seen that the typical time required for persistent entanglement is

$$
\tau \sim \frac{|Z|^{2}}{\lambda_{\mathrm{P}}^{2} \Gamma}
$$

Since $|Z|^{2}=\Gamma^{2}+\Delta_{\mathrm{P}}^{2}$, the increase of detuning for the pumping mode leads to a deceleration of evolution towards the persistent atomic entanglement.

Dynamics of the system described by the effective Hamiltonian in the cavity transparent for Stokes photons was examined in [2]. Comparing the above results with those of [2], one can conclude that the effective model gives only rough picture of purely exponential evolution toward the state (1), completely deleting from the consideration the Rabi oscillations between the levels 1 and 2 . Besides that, the role that detuning for the pumping mode $\Delta_{\mathrm{P}}$ plays in acceleration and deceleration of evolution is lost within the framework of effective model.

Summarizing, we should stress that we have studied the quantum irreversible dynamics of a system of two threelevel $\Lambda$-type atoms, interacting with two modes of quantized electromagnetic field in a cavity under the assumption that Stokes photon either leave cavity freely or is strongly damped.

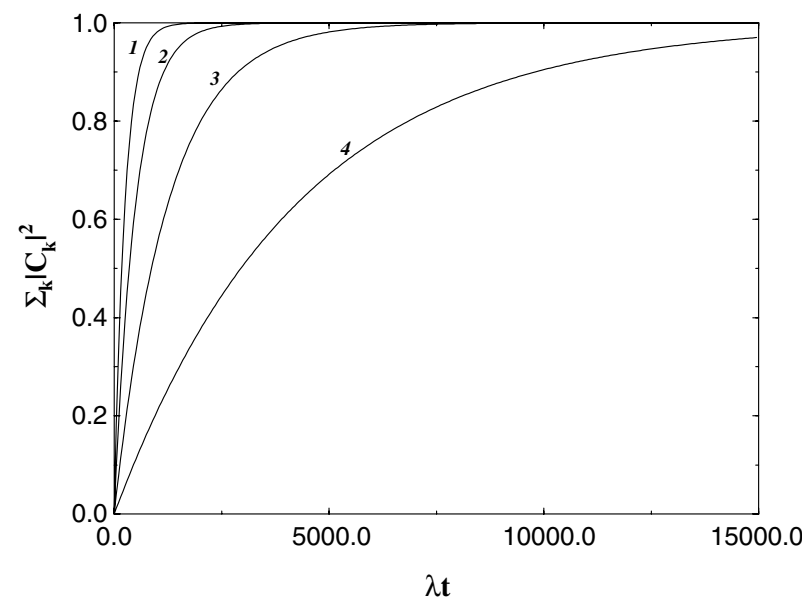

Figure 5. Time evolution of probability (18) to have the persistent entanglement at $\lambda_{\mathrm{P}}=0.001 \Gamma$ for (1) $\Delta_{\mathrm{P}}=0$; (2) $\Delta_{\mathrm{P}}=\Gamma$;

(3) $\Delta_{\mathrm{P}}=2 \Gamma$; (4) $\Delta_{\mathrm{P}}=4 \Gamma$.

It is shown that in both cases system evolves towards the persistent atomic entangled state (1). Such an evolution takes place if the atoms are initially prepared in the ground state, while cavity contains single photon of the pumping mode. It is easily seen that another realistic choice of the initial state, when one of the atoms is excited and cavity field is in the vacuum state, does not lead to evolution towards the persistent entangled state (1).

It is also shown that the effective model with adiabatically removed atomic state 2 predicts correct asymptotic behaviour, while is incapable of description of details of evolution.

Concerning the experimental realization, let us point out that the transitions $1 \leftrightarrow 2$ and $2 \leftrightarrow 3$ should have quite different frequencies. Only in this case it seems to be possible to design a multi-mode cavity with high quality with respect to $\omega_{12}$, permitting either leakage or strong absorption of Stokes photons. An important example is provided by the $3 \mathrm{~S} \leftrightarrow 4 \mathrm{P}$ and $4 \mathrm{P} \leftrightarrow 4 \mathrm{~S}$ transitions in sodium atom and in other alkaline atoms (see [26]). These atoms are widely used in quantum optics, in particular in investigation of Bose-Einstein condensation [27]. The multimode cavities are also used in quantum optics for years (e.g., see [28] and references therein). In particular, the cavities with required properties may be assembled using distributed Bragg reflectors (DBR) and double DBR structures to single out wavelengths corresponding to $\omega_{12}$ and $\omega_{23}$.

The initial state can be prepared in the same way as in [29-31]. First, a single-photon excitation corresponding to the pumping mode is prepared in the cavity. Then, the atoms can propagate through the cavity, using either the same opening or two different but closed openings. The velocity of atoms should be chosen in a proper way, so that the time they spend in the cavity obeys the condition $\tau \gg|Z|^{2} /\left(\lambda_{\mathrm{P}}^{2} \Gamma\right), 1 / \kappa(19)$. All measurements aimed at the detection of atomic entanglement can be performed outside the cavity.

Thus, the realization of persistent entanglement in threelevel $\Lambda$-type atoms seems to be feasible with present experimental technique. It should be emphasized that the single-atom Raman-type process has been observed recently [32]. At the same time, recent progress in investigation of atomic entanglement in the presence of 
dispersive and absorbing objects [33-35] gives a ray of hope to realize persistent atomic entanglement without cavity.

Although our results were obtained for a system of two atoms, they can be generalized to the case of multipartite systems, using the method of [1]. In particular, the persistent atomic entanglement can be obtained in the case of big atomic clusters, containing $2 N$ three-level $\Lambda$-type atoms initially prepared in the ground state and interacting with $N$ pumping photons. Because of the collective processes in atom-photon system, the persistent entanglement should be gained even faster than in the two-atom system. Concerning collective dynamics of Raman-type processes in three-level atoms, see [36] and references therein.

\section{Acknowledgments}

Authors would like to thank Professor A V Andreev, Dr A Beige, Professor V Perinova, Dr A I Lvovsky, Professor $\mathrm{H}$ Walther and Professor D-G Welsch for useful discussions.

\section{References}

[1] Can M A, Klyachko A A and Shumovsky A S 2002 Phys. Rev. A 66022111

[2] Can M A, Klyachko A A and Shumovsky A S 2002 Appl. Phys. Lett. 815072

[3] Can M A, Cakir Ö, Klyachko A A and Shumovsky A S 2003 Phys. Rev. A 68022305

[4] Hagley E, Maitre X, Nogules G, Wunderlich C, Brune M, Raymond J M and Haroshe S 1997 Phys. Rev. Lett. 79

[5] Furusawa A, Sörensen J L, Braunstain S L, Fuchs C A, Kimble H J and Polzik E S 1998 Science 282706

[6] Turchette Q A, Wood C S, King B E, Myatt C J, Liebfried D, Itano W M, Monroe C and Wineland D J 1998 Phys. Rev. Lett. 813631

[7] Bederson B and Walther H 2000 Advances in Atomic, Molecular, and Optical Physics vol 42 (New York: Academic)

[8] Rempe G 2000 Ann. Phys., Lpz. 9843

[9] Raymond J M, Brune M and Haroche S 2001 Rev. Mod. Phys. 73565

[10] Julsgaard B, Kozekhin A and Polzik E 2001 Nature 413400

[11] Plenio M B, Huelga S F, Beige A and Knight P L 1999 Phys. Rev. A 592468
[12] Beige A, Bose S, Braun D, Huelga S F, Knight P L, Plenio M B and Vedral V 2000 J. Mod. Opt. 472583

[13] Solano E, Agarwal G S and Walther H 2002 Preprint quant-ph/0202071

[14] Condon E U and Shortley G H 1987 The Theory of Atomic Spectra (New York: Cambridge University Press)

[15] Moore C E 1971 Atomic Energy Levels (Washington, DC: US National Bureau of Standards)

[16] Yoo H I and Eberly J H 1985 Phys. Rep. 118239

[17] Kien F L and Shumovsky A S 1991 J. Mod. Phys. B 52287

[18] Perina J 1984 Quantum Statistics of Linear and Nonlinear Optical Phenomena (Dordrecht: Reidel)

[19] Gardiner C W and Zoller P 2000 Quantum Noise (Berlin: Springer)

[20] Gerry C C and Eberly J H 1990 Phys. Rev. A 426805

[21] Puri R R, Law C K and Eberly J H 1994 Phys. Rev. A 50 4212

[22] Haroche S and Raymond J M 1985 Advances in Atomic and Molecular Physics vol 20, ed D R Bates and B Bederson (New York: Academic)

[23] Barnett S M and Knight P L 1986 Phys. Rev. A 332444

[24] Dung H T and Shumovsky A S 1992 Phys. Lett. A 169379

[25] Barnett S M and Radmore P 1997 Methods in Theoretical Quantum Optics (Oxford: Clarendon)

[26] NIST Atomic Spectra Database (http://physics.nist.gov/cgi-bin/AtData)

[27] Leggett A J 2001 Rev. Mod. Phys. 73307

[28] Naus H, van Stokkum I H M, Hogervorst W and Ubachs W 2001 Appl. Opt. $\mathbf{4 0} 4416$

[29] Walther H 1997 Quantum Optics and the Spectroscopy of Solids ed T Halioğlu and A S Shumovsky (Dordrecht: Kluwer)

[30] Varcoe B T H, Bratke S, Englert B-G and Walther H 2000 Laser Phys. 101

[31] Walther H 2001 Quantum Communication, Computing, and Measurement ed P Tombesi and O Hirota (New York: Kluwer-Academic)

[32] Hennrich M, Legero T, Kuhn A and Rempe G 2000 Phys. Rev Lett. 854872

[33] Dung H T, Knöll L and Welsch D-G 1998 Phys. Rev. A 57 3931

[34] Dung H T, Scheel S, Welsch D-G and Knöll L 2002 J. Opt. B: Quantum Semiclass. Opt. 4 S169

[35] Welsch D-G, Dung H T and Knöll L 2002 Preprint quant-ph/0205192

[36] Quang T and Shumovsky A S 1990 Interaction of Electromagnetic Field with Condensed Matter ed N N Bogolubov, A S Shumovsky and V I Yukalov (Singapore: World Scientific) 